\title{
The valence-bond charge-transfer-exciton model for predicting nonlinear optical properties (hyperpolarizabilities and saturation length) of polymeric materials
}

\author{
Daqi Lu, Guanhua Chen, ${ }^{\text {a) }}$ and William A. Goddard III) \\ Materials and Molecular Simulation Center, Beckman Institute (139-74), Division of Chemistry and \\ Chemical Engineering (Contribution No. 8766), California Institute of Technology, Pasadena, \\ California 91125
}

(Received 10 August 1993; accepted 6 June 1994)

\begin{abstract}
A simple theory is developed and applied to the polarizability $(\alpha)$, second hyperpolarizability $(\gamma)$, and the saturation lengths for nine polymeric materials. The theory is based on a valence bond view of the ground and excited states and considers the excited states as charge transfer excitons. It involves just two parameters which can be extracted from simple molecular orbital calculations and/or from experimental values of band gap and bandwidth. For the one system (oligothiophenes) with experimental data on saturation length, the predicted behavior of $\alpha$ and $\gamma$ with polymer length is in good agreement with experiment.
\end{abstract}

\section{INTRODUCTION}

Nonlinear optical (NLO) properties are important in numerous applications from lasers to optical switches and electronics. ${ }^{1}$ Some of the best NLO properties are displayed by organic materials, for example charge-transfer type organic molecules where contributions to NLO properties come mostly from charge resonance between the donor and acceptor. $^{2}$ However, for molecules with a long polyene bridge, the bridge can also contribute significantly, leading to even better nonlinear optical properties. Thus Marder and co-workers ${ }^{3}$ have shown that crystals of (1) or (2) lead to $y=10^{-34} \mathrm{esu}$,
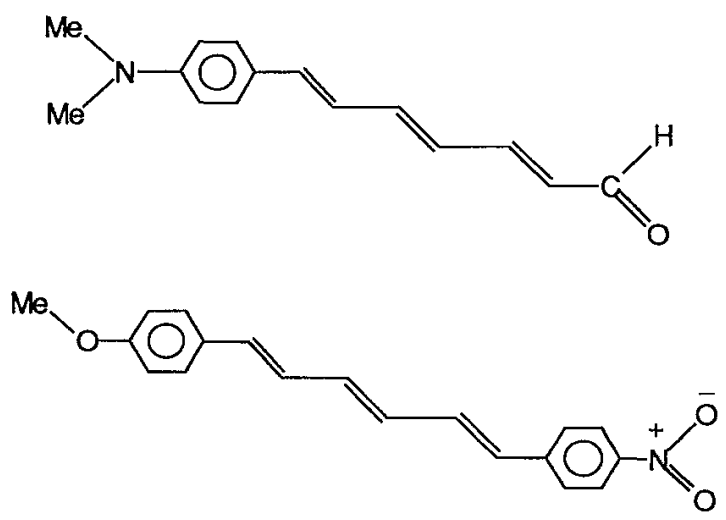

Although the hyperpolarizability increases with the increasing length of the polymer bridge, experiments ${ }^{4}$ indicate that the $\gamma$ value saturates (becomes linear in chain length) near 7 units for oligothiophenes

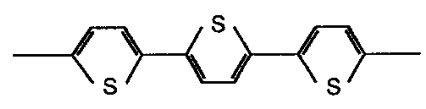

${ }^{a)}$ Current address: Department of Chemistry, University of Rochester, Rochester, New York.

${ }^{b)}$ To whom correspondence should be addressed.
In order to design and optimize such materials, it is of interest to predict the NLO properties as a function of donor and acceptor, as a function of the nature and length of the polymer bridge, and as a function of solvent.

There have been many recent theoretical ${ }^{5-8}$ and experimental ${ }^{3}$ studies directed at understanding and optimizing the NLO properties of organic compounds. The conventional methods of calculating hyperpolarizabilities are (i) calculating the occupied and excited molecular orbitals of a molecule and summing over the excited states," Eqs. (36)-(38); (ii) using finite-fields and taking energy differences. $^{21-22}$

These methods work well for small molecules; however, for large and complex molecules, such methods rapidly become extremely tedious and compute intensive.

We develop here an alternate approach based on a valence bond view of the bonding and a charge-transfer-exciton view of the excited states. We use the finite-field method to get the expression of the polarizability and hyperpolarizability. This method allows very rapid calculations and leads to results in good agreement with experiment. With an additional approximation, it leads to analytical formulas that provide physical insight into the factors determining the magnitude of $\alpha$ and $\gamma$ and the saturation length.

\section{THE VALENCE-BOND CHARGE-TRANSFER- EXCITON (VB-CTE) MODEL OF POLYMER EXCITED STATES}

\section{A. The Hamiltonian}

As a prototype we will consider a rigid-chain polymer with $N$ monomers (taking $N$ to be odd for convenience). We assume that the most important states of the polymer can be described with a valence bond (VB) description using only the highest-occupied molecular orbital (HOMO) and the lowest-unoccupied molecular orbital (LUMO) of each monomer unit. In the ground state the HOMO is doubly occupied 
and the LUMO is empty. Now consider the excitation of a monomer (site $p$ ) near the middle of a long polymer. The occupied LUMO may be on the same monomer (leading to a covalent excitation energy, $\Delta E_{c}$ ), on adjacent monomers (sites $p \pm 1$ ), or on sites farther away (sites $p \pm 2, p \pm 3$, etc.).
We let $\phi_{p q}$ denote the many-electron charge-transfer-exciton wave function in which an electron at site $p$ is excited to a monomer $q$ units to the right of $p$, leaving a hole at site $p$. Considering a fixed site $p$ for the hole, the Hamiltonian matrix between various $\phi_{p q}$ becomes

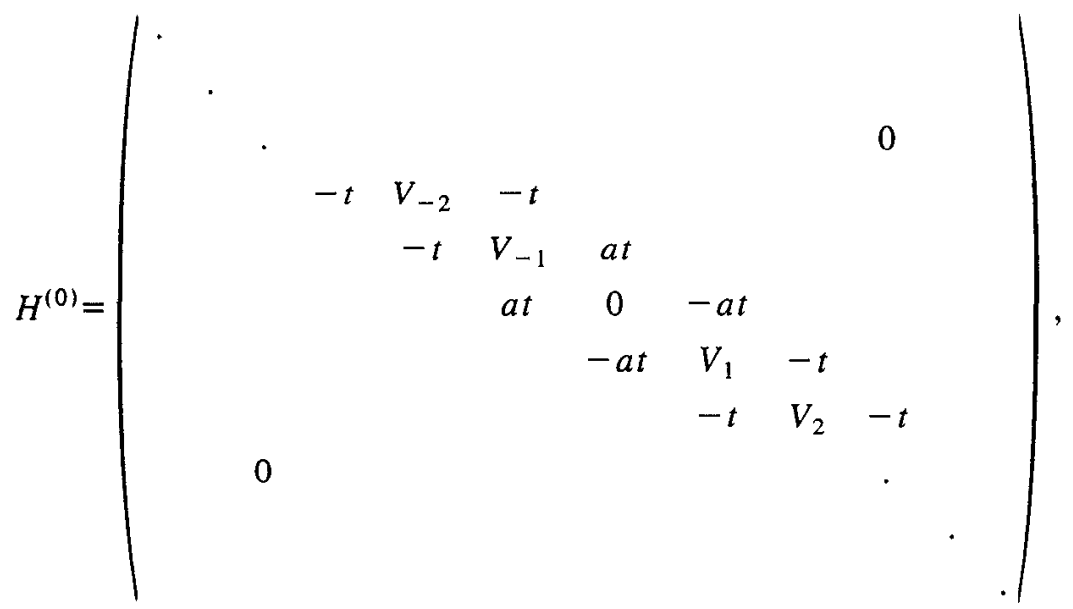

where matrix elements are kept only between adjacent sites.

The diagonal matrix element $V_{q}$ is the energy required to remove an electron from the HOMO of one monomer and place it in the LUMO of the monomer $q$ units away. It becomes

$$
V_{q}=\left\langle\phi_{p q}|\not{F}| \phi_{p q}\right\rangle=\mathrm{IP}-\mathrm{EA}-\frac{e^{2}}{\epsilon|q| R_{0}},
$$

where IP is the ionization potential of the monomer, EA is the electron affinity of the monomer, $R_{0}$ is the distance between the nearest monomer centers, and $q$ is the number of monomers between the donor and acceptor states $(q= \pm 1$, \pm 2 , etc.).

The transfer matrix elements are (assuming a symmetric monomer)

$$
\begin{aligned}
& -t=\left\langle\phi_{p q}|\not{X}| \phi_{p, q+1}\right\rangle \quad \text { if }|q|>1, \\
& -a t=\left\langle\phi_{p 0}|\mathscr{X}| \phi_{p 1}\right\rangle \quad \text { if } q=1, \\
& a t=\left\langle\phi_{p,-1}|\not{X}| \phi_{p 0}\right\rangle \quad \text { if } q=-1,
\end{aligned}
$$

where the opposite sign of the matrix elements for $q= \pm 1$ arises from the antisymmetry between the LUMO and HOMO (see Appendix A and Fig. 4). We have ignored the covalent excitation at $q=0$ because it does not contribute to changes in the dipole moment.

The evaluation of $t$ and $a$ are discussed in Appendix A where we find that for the butadiene unit of thiophene

$$
\begin{aligned}
& t=0.36 \beta, \\
& a=\sqrt{2},
\end{aligned}
$$

where $\beta$ is the resonance integral between the adjacent $s p^{2}$ carbon orbitals.

\section{B. Dipole moment}

Writing the dipole operator as $\mu=\Sigma_{l} q_{l} R_{l}$, where $q_{l}$ is the charge on monomer $l$, the Hamiltonian in an electric field $\mathscr{E}$ becomes

$$
H=H^{0}-\mu \cdot \mathscr{E} \text {. }
$$

In order to examine the polarizability and hyperpolarizability, we need matrix elements of the dipole operators,

$$
P_{i j, \alpha}=\left\langle\phi_{p i}\left|\mu_{\alpha}\right| \phi_{p j}\right\rangle
$$

where $\alpha$ is the component $(x, y$, or $z)$. Assuming that only the component along the polymer axis (say $z$ ) is important, Eq. (11) becomes

$$
P_{i j, z}=\left\langle\phi_{p i}\left|\sum_{l} q_{l} R_{l}\right| \phi_{p j}\right\rangle=\delta_{i j}\left(-e R_{i}\right)
$$




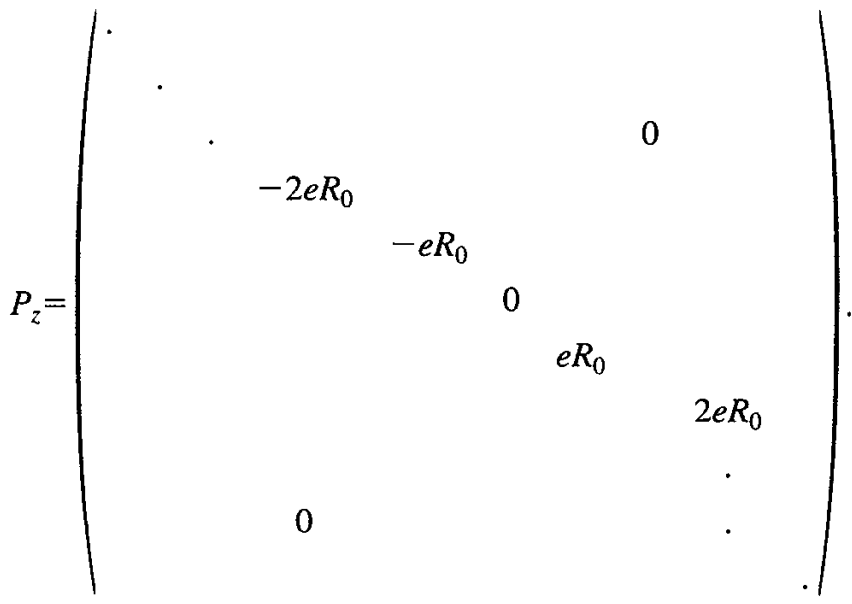

(13)

For a finite electric field $\mathscr{E}$, the energy of the ground state is

$$
E^{0}=\left\langle 0\left|H^{0}-P \cdot \varnothing\right| 0\right\rangle
$$

leading to static polarizability and hyperpolarizabilities given by

$$
\begin{aligned}
& \alpha_{z z}=-\frac{d^{2} E^{0}}{d \mathscr{C}_{z}^{2}}, \\
& \beta_{z z z}=-\frac{1}{2} \frac{d^{3} E^{0}}{d \mathscr{E}_{z}^{3}}, \\
& \gamma_{z z z z}=-\frac{1}{6} \frac{d^{4} E^{0}}{d \mathscr{E}_{z}^{4}} .
\end{aligned}
$$

The above analysis is for a fixed site $p$. Summing over all $p$ leads to total values approximately $N$ times as large.

\section{Neglect of Coulomb interactions}

Although the Coulomb correction in Eq. (5) may be large, we will first consider the simple approximation where these terms are ignored, leading to

$$
V_{q}^{N_{0} Q}=V=\text { IP-EA. }
$$

The Hamiltonian in a electric field $H=H^{0}-P \cdot \%$ can be rewritten as $H=H_{0}+W$, where $H_{0}$, Eq. (19), is the unperturbed Hamiltonian matrix and $W$, Eq. (20), is considered as the perturbation Hamiltonian matrix,

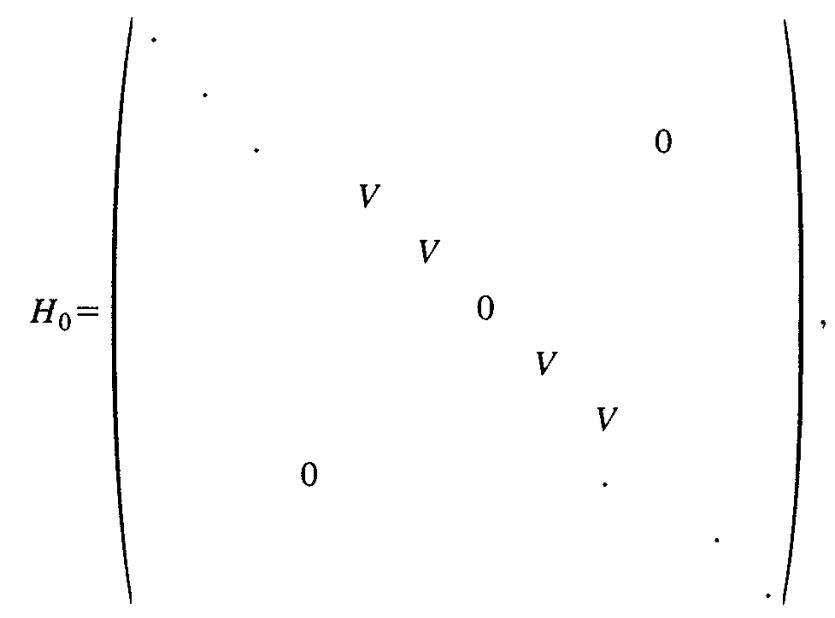

$$
\gamma_{z z z z}=\frac{8 a^{2} e^{4} R_{0}^{4} \eta^{2}}{V^{3}} G(\eta)
$$

$$
\begin{aligned}
& \alpha_{z z}=\frac{4 a^{2} e^{2} R_{0}^{2} \eta^{2}}{V} A(\eta), \\
& \beta_{z z z}=0,
\end{aligned}
$$

where

$$
A(\eta)=\sum_{i=0}^{\infty} A_{2 i} \eta^{2 i}
$$




$$
G(\eta)=\sum_{i=0}^{\infty} G_{2 i} \eta^{2 i}
$$

and

$$
\eta=\frac{t}{V}
$$

is the dimensionless energy dominating the saturation length. Because the monomers were assumed symmetric in Eqs. (6) and (7), the $\beta_{z z z}$ is zero.

As shown in Appendix $C, t$ is related to the bandwidth (B) by

$$
B \approx 4 t,
$$

while $V$ is related to the energy gap $\left(E_{g}\right)$ by

$$
E_{g} \approx V-2 t \text {. }
$$

Thus

$$
\eta=\frac{t}{V} \approx \frac{\frac{B}{4}}{E_{g}+\frac{B}{2}} .
$$

We find that large $t$ leads to a larger saturation length while large $V$ (or band gap) leads to a a smaller saturation length. For $E_{g}=0$, Eq. (29) leads to $\eta=\frac{1}{2}$. Thus the range of $\eta$ is 0 to $\frac{1}{2}$.

\section{SATURATION LENGTH}

\section{A. Empirical formula for saturation lengths}

As shown in Appendix A, $a \sim \sqrt{2}$, and hence the polynomials in Eqs. (24) and (25) are functions of only one parameter, $\eta=(t / V)$. Consequently the saturation length is a function only of $\eta=(t / V)$. The length dependence of $\alpha$ and $\gamma$ are shown for various values of $\eta$ in Fig. 1 (scaled by the limiting values). We will define the saturation length $L_{\alpha}$ and $L_{\gamma}$ as the number of monomers for which $\alpha$ and $\gamma$, respectively, attain $95 \%$ of the limiting value. To a good approximation we find [Fig. 1(b)] that

$$
L_{\gamma} \sim 1+38 \frac{t}{V}
$$

for $\eta=0.1-0.4$. Similarly the saturation length for polarizability is approximated by

$$
L_{\alpha} \sim 1+20 \frac{t}{V}
$$

as shown in Fig. 1(a). Combining Eqs. (30) and (31) lead to

$$
L_{\gamma} \sim 1.9 L_{\alpha}-0.9
$$

\section{B. Effects of Coulomb interaction and frequency of electromagnetic fields}

The results in Sec. II C and Sec. III B are approximate since the Coulomb interaction in Eq. (5) is ignored. Includ-
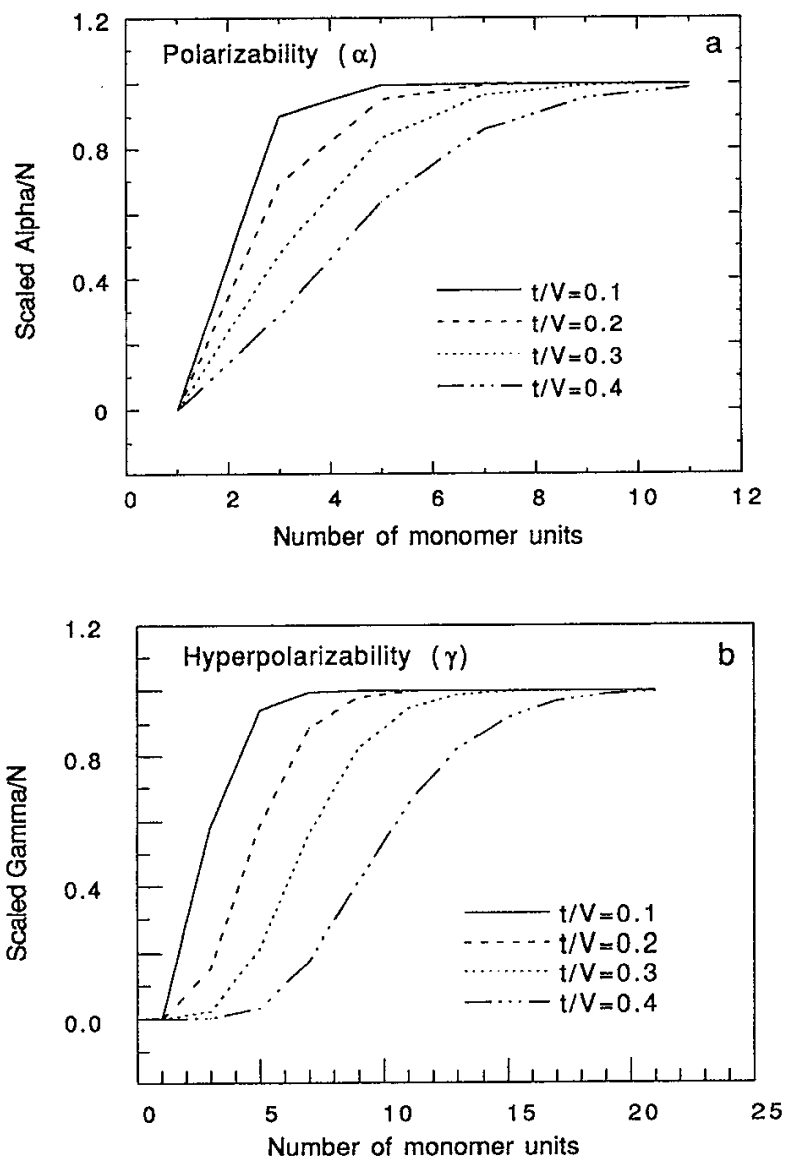

FIG. 1. Dependence of saturation behavior on $\eta=t / V$, using Eqs. (21) and (23) in the $N o Q$ approximation. (a) Polarizability and (b) hyperpolarizability.

ing the Coulomb interactions between electrons and holes [using Eq. (5) instead of Eq. (18)], prevents us from obtaining analytical results as in Sec. II C. Also for frequency dependence polarizabilities we again do not get analytical formulas.

For the general case we use sum-over-state approach in which the polarizability and hyperpolarizability have the form

$$
\alpha_{i j}(-\omega, \omega)=2 I_{-\omega, \omega}\left(\frac{e^{2}}{\hbar}\right) \sum_{n} \frac{r_{g n}^{i} r_{n g}^{j}}{\omega_{n g}-\omega},
$$

$$
\begin{aligned}
\beta_{i j k}\left(-\omega_{\sigma} ; \omega_{1}, \omega_{2}\right)= & 3 K\left(-\omega_{\sigma} ; \omega_{1}, \omega_{2}\right)\left(\frac{e^{3}}{\hbar^{2}}\right) I_{-\sigma ; 1,2} \\
& \times \sum_{m, n} \prime\left[\frac{r_{g n}^{i} \bar{r}_{n m}^{j} r_{m g}^{k}}{\left(\omega_{m g}-\omega_{\sigma}\right)\left(\omega_{n g}-\omega_{1}\right)}\right],
\end{aligned}
$$


TABLE I. Calculated properties of polyacetylene as a function of polymer length, $N$, using butadiene as the monomer (thus the number of double bonds is $2 N) . Q$ indicates the use of Eq. (5) with ${ }^{2} \epsilon=2$ and $V=2.85 \mathrm{eV}$, whereas $N o Q$ indicates the use of Eq. (18). All cases use zero frequency.

\begin{tabular}{cccccc}
\hline \hline & $\begin{array}{c}\text { Excitation } \\
N\end{array}$ & \multicolumn{2}{c}{$\langle\alpha\rangle^{\mathrm{c}}\left(10^{-23} \mathrm{esu}\right)$} & \multicolumn{2}{c}{$\langle\gamma\rangle^{\mathrm{d}}\left(10^{-34} \mathrm{esu}\right)$} \\
\cline { 3 - 6 } nnergy $^{\mathrm{b}}(\mathrm{eV})$ & $N o Q$ & $Q$ & $N o Q$ & $Q$ \\
\hline 3 & 2.47 & 3.20 & 8.38 & 3.10 & 5.56 \\
5 & 2.00 & 8.39 & 22.83 & 30.51 & 75.07 \\
7 & 1.86 & 12.98 & 35.62 & 84.90 & 236.01 \\
9 & 1.82 & 16.97 & 46.61 & 136.78 & 385.49 \\
11 & 1.81 & 20.79 & 57.09 & 177.73 & 498.88 \\
13 & 1.80 & 24.58 & 67.48 & 212.91 & 596.08 \\
15 & 1.80 & 28.36 & 77.87 & 246.28 & 688.98 \\
17 & 1.80 & 32.15 & 88.25 & 279.23 & 781.02 \\
\hline \hline
\end{tabular}

${ }^{\mathrm{a} S e e}$ Ref. 12.

${ }^{\mathrm{b}}$ The value of $V=2.85 \mathrm{eV}$ was obtained by requiring that $E_{g}=1.80 \mathrm{eV}$ for $N=\infty$.

$\langle\alpha\rangle\rangle=\frac{1}{3}\left\langle\alpha_{i z}\right\rangle$.

$\mathrm{d}\langle\gamma\rangle=\frac{1}{5}\left\langle\gamma_{z z z z}\right\rangle$.

$$
\begin{aligned}
\gamma_{i j k l}( & \left.-\omega_{\sigma} ; \omega_{1}, \omega_{2}, \omega_{3}\right) \\
= & 4 K\left(-\omega_{\sigma} ; \omega_{1}, \omega_{2}, \omega_{3}\right)\left(\frac{e^{4}}{\hbar^{3}}\right) I_{-\sigma ; 1,2,3} \\
& \times\left[\sum_{m, n, p}, \frac{r_{g p}^{i} r_{p n}^{j} \bar{r}_{n m}^{k} r_{m g}^{l}}{\left(\omega_{p g}-w_{\sigma}\right)\left(\omega_{n g}-\omega_{1}-\omega_{2}\right)\left(\omega_{m g}-\omega_{1}\right)}\right. \\
& -\sum_{m n}, \frac{r_{g m}^{i} r_{m g}^{j} r_{g n}^{k} r_{n g}^{l}}{\left(\omega_{m g}-\omega_{\sigma}\right)\left(\omega_{n g}-\omega_{1}\right)\left(\omega_{n g}+\omega_{2}\right)} .
\end{aligned}
$$

Here,

(i) $g$ indicates the ground state and $\Sigma^{\prime}$ indicates that $g$ is excluded from the sum.

(ii) $\bar{r}_{k l}^{i}=r_{k l}^{i}-r_{g g}^{i}$, where $r_{k l}^{i}$ is the dipole matrix element for component $i$ between states $l$ and $k$.

(iii) $\omega_{\sigma}=\Sigma_{i} \omega_{i}$. (iv) $K\left(-\omega_{\sigma} ; \omega_{1}, \omega_{2}, \omega_{3}\right)$ is a numerical factor determined by the nature of the NLO process. ${ }^{9}$

(v) $I_{-\sigma, 1,2,3}$ denotes the average of all terms generated by permuting $\sigma, \omega_{1}, \omega_{2}, \omega_{3}$.

The excitation energies are obtained by diagonalizing the Hamiltonian matrix (4), and the dipole matrix elements can be obtained by transforming matrix (13) to the eigenstates. The resulting values of $\alpha$ and $y$ are listed in Tables I and II for polyacetylene and oligothiophene, respectively. These tables show that Coulomb interactions do affect the magnitude of $\alpha$ and $\gamma$. Thus including Coulomb interactions makes $\alpha_{z z} 2$ to 3 times larger and makes $\gamma_{z z z z} 2$ to 3 times larger. However, as shown in Fig. 2, the saturation behavior is nearly identical. The reason is that at the point where the separation between the electron and hole is approximately the saturation length, the Coulomb interaction is much less than $V$, leading to negligible effect on exciton size.

The frequency also affects the magnitude and saturation length for $\alpha$ and $\gamma$. When the frequency is near resonance, the magnitude and saturation length change significantly. Thus from Table II, the magnitude of $\gamma_{z, z z z}$ increases by a factor of 10 . This is because $\omega=1.16 \mathrm{eV}$ is near the resonance frequency of $\omega=1.4 \mathrm{eV}$. The saturation length also increases by 2 units [Fig. 3(b)]. However for frequencies far from resonance, the change in saturation length is negligible (Fig. 3), and the change in magnitude is about one order of magnitude.

\section{CALCULATIONS OF THE SATURATION LENGTHS FOR OLIGOTHIOPHENES AND POLYACETYLENE}

For oligothiophenes, the saturation length of $\alpha$ and $\gamma$ with chain length has recently been observed experimentally. ${ }^{4}$ We will use the above theory to predict the saturation properties of polyacetylene and oligothiophenes.

\begin{tabular}{|c|c|c|c|c|c|c|c|c|c|c|}
\hline \multirow[b]{3}{*}{$N$} & \multicolumn{2}{|c|}{$\begin{array}{c}\text { Excitation } \\
\text { nergies }(\mathrm{eV})\end{array}$} & \multicolumn{3}{|c|}{$\left\langle\alpha_{z z}\left(10^{-23} \mathrm{esu}\right)\right\rangle$} & \multirow{3}{*}{ Expt. $^{a}$} & \multirow{2}{*}{\multicolumn{3}{|c|}{$\left\langle\gamma_{z z z z}\left(10^{-34} \mathrm{esu}\right)\right\rangle$}} & \multirow{3}{*}{ Expt. ${ }^{b}$} \\
\hline & \multirow[b]{2}{*}{ Calc. $^{c}$} & \multirow[b]{2}{*}{ Expt. } & \multicolumn{3}{|c|}{ Calculation } & & & & & \\
\hline & & & $\begin{array}{c}N_{0} Q \\
\omega=0\end{array}$ & $\begin{array}{c}Q \\
\omega=0\end{array}$ & $\begin{array}{c}Q \\
\omega=1.95\end{array}$ & & $\begin{array}{l}N_{0} Q \\
\omega=0\end{array}$ & $\begin{array}{c}Q \\
\omega=0\end{array}$ & $\begin{array}{c}Q \\
\omega=1.16\end{array}$ & \\
\hline 3 & 3.52 & 3.67 & 1.25 & 2.71 & 3.90 & 1.1 & 0.76 & 2.10 & 5.39 & 9.9 \\
\hline 5 & 2.99 & 3.01 & 2.82 & 6.36 & 10.93 & 2.6 & 4.37 & 12.8 & 60.3 & 107 \\
\hline 7 & 2.84 & 2.81 & 4.10 & 9.35 & 17.13 & 6.6 & 8.76 & 26.5 & 188.4 & 360 \\
\hline 9 & 2.79 & 2.78 & 5.30 & 12.09 & 22.45 & $\ldots$ & 12.20 & 37.11 & 321.8 & 370 \\
\hline 11 & 2.77 & 2.77 & 6.48 & 14.78 & 27.51 & 7.0 & 15.11 & 45.98 & 429.2 & 460 \\
\hline 13 & 2.77 & $\cdots$ & 7.66 & 17.46 & 32.52 & $\cdots$ & 17.88 & 54.43 & 519.3 & $\cdots$ \\
\hline 15 & 2.77 & $\cdots$ & 8.84 & 20.15 & 37.53 & $\cdots$ & 20.64 & 62.81 & 602.5 & $\cdots$ \\
\hline
\end{tabular}

TABLE II. Experimental and theoretical properties of oligothiophenes as a function of polymer length, $N$. Notation is the same as for Table I except ${ }^{\mathrm{a}} \epsilon=2.5 . \omega=0$ indicates the static case, whereas $\omega \neq 0$ indicates finite frequency; ${ }^{\mathrm{a}} \omega=1.95 \mathrm{eV}$ for $\langle\alpha(-\omega ; \omega)\rangle$ and $\omega=1.16 \mathrm{eV}$ for $\langle\chi(-2 \omega ; \omega, \omega, 0)\rangle$.

${ }^{a}$ See Ref. 18.

${ }^{b}$ See Ref, 4.

cThe value of $V=4.09 \mathrm{eV}$ was obtained by requiring that $E_{g}=2.77 \mathrm{eV}$ for $N=\infty ; t=0.83 \mathrm{eV}$ and $a=\sqrt{2}$ are derived in Appendix A. 

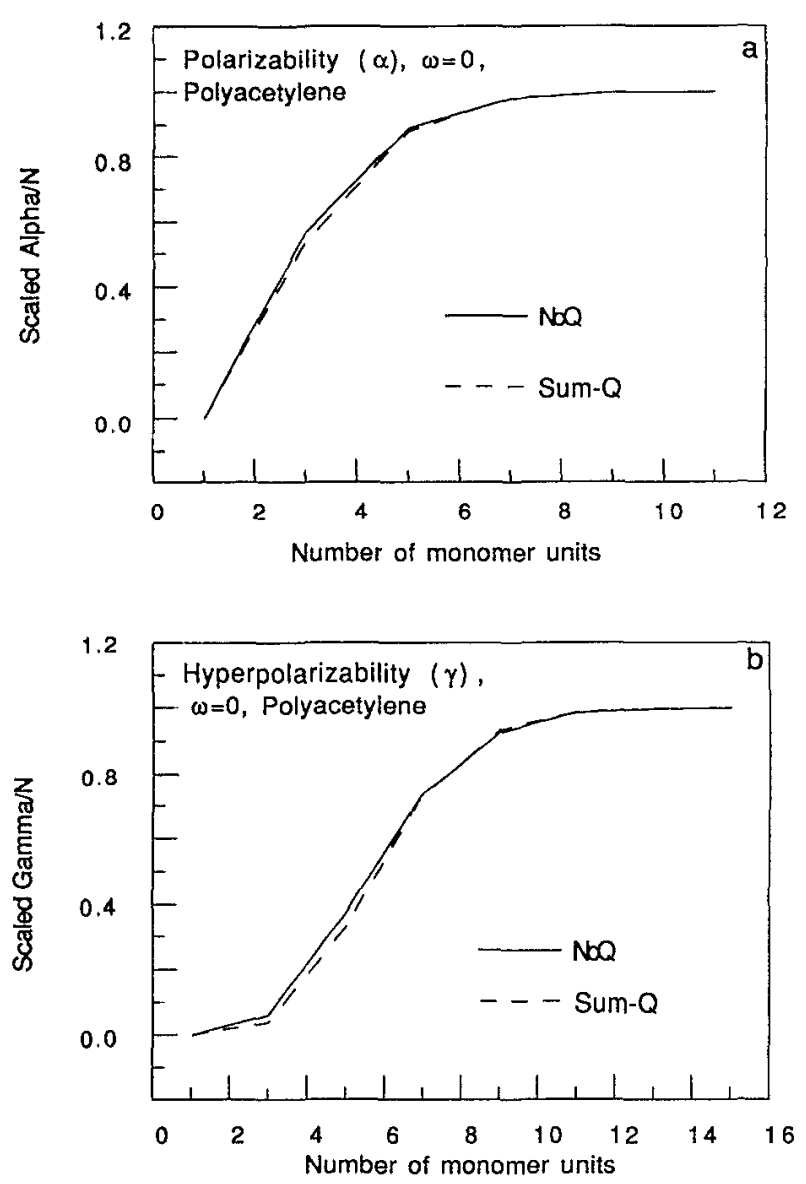

FIG. 2. The $\omega=0$ saturation behavior of polyacetylene for (a) polarizability and (b) hyperpolarizability. The dashed line $(N o Q)$ uses sum-over-state withrut Coulomb interaction (perturbation theory leads to essentially identical results). The solid line (Sum- $Q$ ) uses sum-over-state with Coulomb interaction.

\section{A. Oligothiophenes, comparison to experiment}

To estimate the NLO properties of oligothiophenes, we estimated $a$ and $t$ by ignoring the sulfur and using Hückel theory (MO's for butadiene in Fig. 4). The analysis in Appendix A leads to

$$
t=0.36 \beta \text {. }
$$

A fit to the band states of polyacetylene ${ }^{12}$ gives

$$
\beta=2.4(1.0 \pm 0.7 \delta) \mathrm{eV},
$$

where $\delta$ is the bond length alternation (half the difference in bond length between double and single bonds). Butadiene leads to $2 \delta=(1.47-1.34)=0.13 \AA$, and hence we take

$$
\delta=0.065 \AA \text {. }
$$

With Eqs. (38) and (8), Eq. (37) leads to

$$
t=0.83 \mathrm{eV} \text {. }
$$

We solved for the eigenvalues of Eq. (4) as a function of chain length (see Table II) and find that

$$
V=4.09 \mathrm{eV}
$$
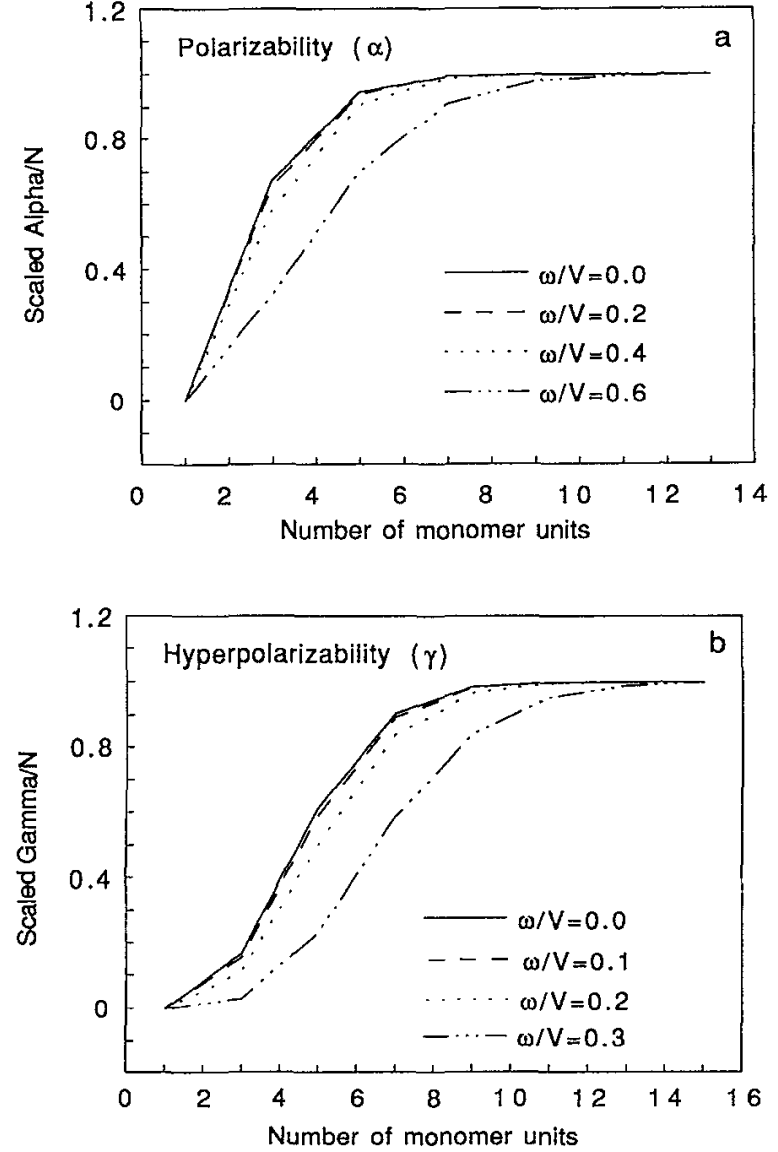

FIG. 3. Effect of frequency on the saturation behavior of oligothiophene. (a) Polarizability and (b) hyperpolarizability. The resonance frequency of $\alpha$ is $2.77 \mathrm{eV}$ (corresponding to $\omega / V=0.67$ ) whereas the resonance frequency of $\gamma$ is $1.39 \mathrm{eV}$ (corresponding to $\omega / V=0.34$ ).

leads to the experimental energy gap $^{4}\left(E_{g}=2.77 \mathrm{eV}\right.$ for saturation). Indeed we obtain an excellent fit with the observed transitions for shorter polymers. From Eq. (C6),

$$
V \approx E_{g}+2 t
$$

which would lead to $V=2.77+1.66=4.43 \mathrm{eV}$, in reasonable agreement with the more exact calculation of $4.09 \mathrm{eV}$. [Indeed Eq. (C8) leads to $V=4.09$, in excellent agreement.] Equations (39) and (40) lead to $t / V=0.20$, suggesting saturation lengths of $L_{\alpha}=5$ monomers and $L_{\gamma}=8.6$ monomers, which are in excellent agreement with the experiment $L_{\alpha} \sim 5$ and $L_{\gamma} \sim 8 .{ }^{4}$ Using the above parameters and including Coulomb interactions, leads to the $\alpha$ and $\gamma$ in Fig. 5 (using experimental ${ }^{4}$ frequencies). The results are in excellent agreement with experiment except for $N=7$. Experimentally $\alpha / N$ and $\gamma / N$ for $N=7$ are greater than the values for larger $N$ whereas calculations show that $\alpha / N$ and $\gamma / N$ increase monotonically with $N$. Consequently we suggest there may be an experimental artifact at $N=7$.

The $w=0$ values for $\alpha$ and $\gamma$ calculated from formulas (24) and (25) and from the sum-over-state (33) and (35) are listed for comparison in Table II. The calculated $y$ at $w=1.16$ 

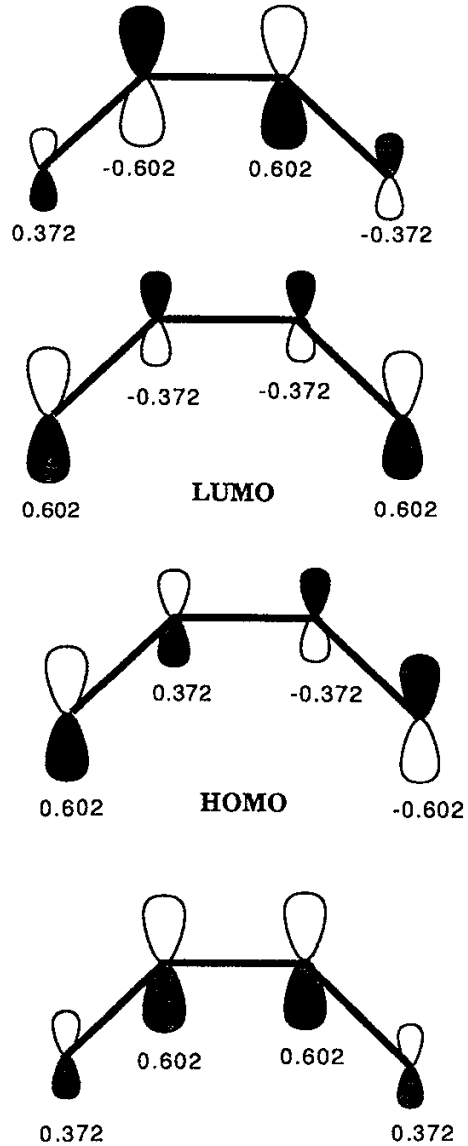

FIG. 4. The Hückel molecular orbitals of butadiene. Thus $\quad \theta^{\text {UMMO }}=0.602 \chi_{1}+0.372 \chi_{2}-0.372 \chi_{3}-0.602 \chi_{4} \quad$ and $\theta^{\mathrm{HOMO}}=0.602 \chi_{1}-0.372 \chi_{2}-0.372 \chi_{3}+0.602 \chi_{4}$, where $\chi_{i}$ are the four atomic orbitals.

$\mathrm{eV}$ is off from the experiment by $6 \%$ for $N=11$ and by $13 \%$ for $N=9$. However the calculated $\alpha$ at $\omega=1.95 \mathrm{eV}$ is about 3 to 4 times the observed values.

\section{B. Polyacetylene}

Next we consider the case of a simple trans polyene (polyacetylene). Using butadiene as the monomer leads to

$$
\begin{aligned}
& t=0.83 \mathrm{eV}, \\
& a=\sqrt{2},
\end{aligned}
$$

just as for oligothiophenes.

We solved for the eigenvalues of matrix (4) as a function of chain length and chose

$$
V=2.85 \mathrm{eV} \text {, }
$$

so that excitation energy for large $N$ equals the experimental band gap, $E_{g}=1.8 \mathrm{eV}$. In comparison, use of Eq. (41) with the observed gap leads to $V=3.47 \mathrm{eV}$ while use of Eq. (C9) from Appendix $C$ leads to $V=2.88$, in excellent agreement with Eq. (44).

Equations (42) and (44) lead to $t / V=0.29$ which from Eqs. (30) and (31) suggest $L_{\alpha} \approx 6.8$ and $L_{\gamma} \approx 12$ butadiene units (13.6 and 24 ethylene units), respectively. That means that saturation length of $\alpha$ is about 28 carbon atoms and the
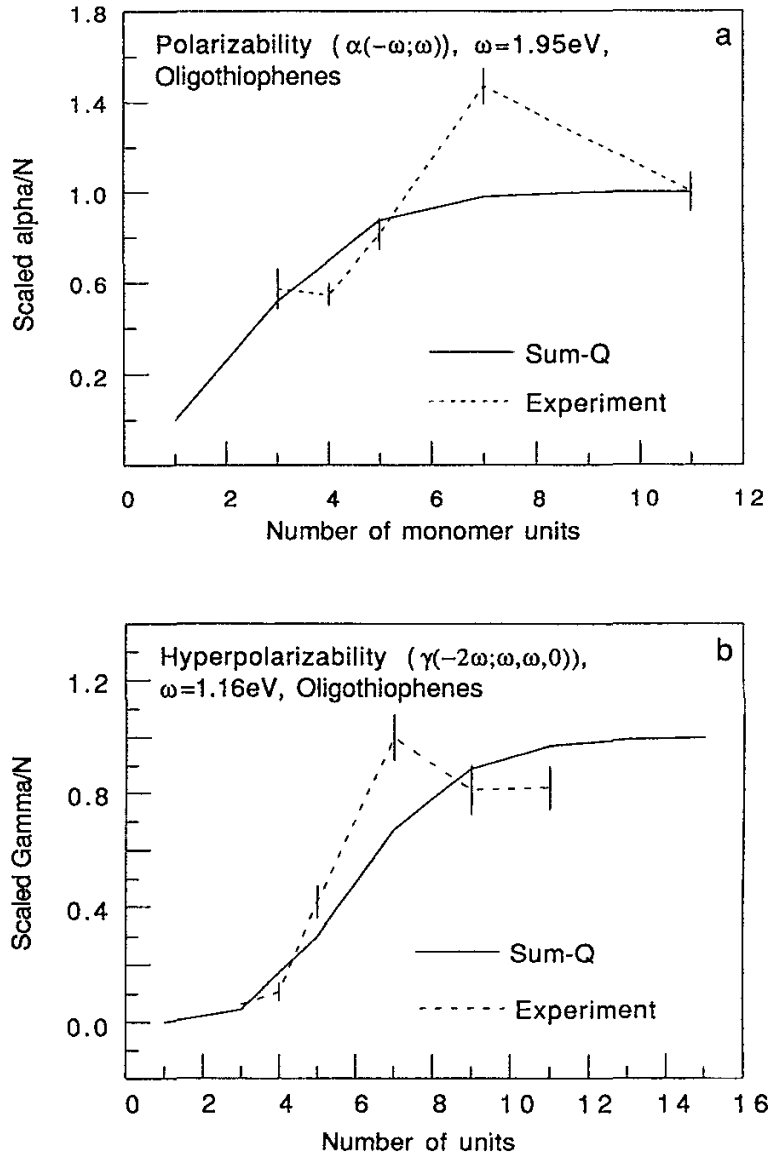

FIG. 5. Comparison of theory and experiment (Ref. 4) for the saturation behavior of oligothiophenes. (a) Polarizability $(\omega=1.95 \mathrm{eV})$ and (b) hyperpolarizability $(\omega=1.16 \mathrm{eV})$. The dashed line connects experimental data (with error bars). The solid line connects theoretical predictions based on sum-over-states with Coulomb interactions.

saturation length for $\gamma$ is about 48 carbon atoms. This result is in agreement with other theoretical results (21 double bonds for $\gamma) .{ }^{14}$ The saturation length for $\gamma$ of oligothiophenes is 16 double bonds, considerably shorter than that of polyacetylene. This results primarily from the higher value of $V$ (and hence higher band gap) arising from the sulfur in oligothiophenes.

\section{DISCUSSION}

\section{A. Comparison with experiment}

Considering the simplicity of this VB-CTE model, the predicted results are excellent. Important approximations are the following:

(i) This model is derived for the long chain limit and should not hold for short chain lengths.

(ii) The hole is assumed fixed.

(iii) For nearest neighbor interactions, the expressions for Coulomb interaction should be corrected for shielding.

(iv) The covalent (same site) exciton is neglected.

(v) The correlation between electron-hole pairs is neglected. 
TABLE III. Predicted saturated values polarizability $(\alpha)$, hyperpolarizability $(\gamma)$, and saturation lengths $\left(L_{\alpha}, L_{\gamma}\right)$ of polymers. Based on Eqs. (21) and (23) with $\omega=0$.

\begin{tabular}{|c|c|c|c|c|c|c|c|c|c|c|}
\hline Polymer & Monomer $^{f}$ & $\begin{array}{c}B^{\mathrm{a}} \\
(\mathrm{eV})\end{array}$ & $\begin{array}{c}E_{g}^{\mathrm{b}} \\
(\mathrm{eV})\end{array}$ & $\begin{array}{c}t^{\mathrm{g}} \\
(\mathrm{eV})\end{array}$ & $\begin{array}{c}V^{\mathrm{g}} \\
(\mathrm{eV})\end{array}$ & $\eta$ & $L_{\alpha}^{\mathrm{c}}$ & $L_{y}^{c}$ & $\begin{array}{c}\alpha_{z z}^{\mathrm{d} \cdot \mathrm{e}} / N \\
\left(10^{-23} \mathrm{esu}\right)\end{array}$ & $\begin{array}{c}\gamma_{z z z z}^{\mathrm{d}, \mathrm{e}} / N \\
\left(10^{-34} \mathrm{esu}\right)\end{array}$ \\
\hline Polydiacetylene (acetylenic) & $a$ & 3.9 & 2.1 & 1.0 & 4.1 & 0.24 & 5.8 & 10.1 & 2.41 & 11.30 \\
\hline Polyparaphenylene vinylene & $c$ & 2.8 & 3 & 0.7 & 4.4 & 0.16 & 4.2 & 7.1 & 2.49 & 15.93 \\
\hline Polypyrrole & $d$ & 3.8 & 3.2 & 0.95 & 5.1 & 0.19 & 4.8 & 8.2 & 1.13 & 2.55 \\
\hline Polythiophene vinylene & $e$ & 2.5 & 2.2 & 0.6 & 3.4 & 0.18 & 4.6 & 7.8 & 3.88 & 44.90 \\
\hline Polybenzothiophene & $h$ & 1.3 & $3.1^{\mathrm{a}}$ & 0.3 & 3.8 & 0.08 & 2.6 & 4.0 & 1.09 & 3.45 \\
\hline Oliothiophenes & $j$ & $\cdots$ & 2.77 & $0.83^{h}$ & 4.09 & 0.20 & 5.0 & 9.0 & 1.77 & 6.87 \\
\hline Polyacetylene (trans) & $i$ & $\ldots$ & 1.8 & $0.83^{\mathrm{h}}$ & 2.85 & 0.29 & $6.8^{\mathrm{i}}$ & $12.0^{\mathrm{i}}$ & 5.65 & 82.06 \\
\hline
\end{tabular}

${ }^{2}$ Calculated values from $\operatorname{Ref}, 15$.

Experimental values from Ref. 15.

'Calculated using Eqs. (30) or (31).

${ }^{\mathrm{d}}$ Using $a=\sqrt{2}$.

'Values at saturation using Eqs. (21) and (23) and $\omega=0$. An approximate correction for finite frequency is to multiply $\alpha(-\omega, \omega)$ by $E_{g} /\left(E_{g}-\omega\right)$ and to multiply $x-3 \omega ; \omega, \omega, \omega)$ by $E_{g}^{3} /\left(E_{g}-\omega\right)\left(E_{g}-2 \omega\right)\left(E_{g}-3 \omega\right)$.

'See Fig. 6.

Esing Eqs. (27) and (28) unless otherwise noted.

"Derived in text rather than from $B$.

'The entries in the table are in terms of butadiene monomers. The number of double bonds is 14 for $\alpha$ and 24 for $\gamma$.

\section{B. Predictions for other materials, design considerations}

The analytical results (21)-(29) (obtained for a static external field with neglect of Coulomb interactions), indicate that the magnitudes of $\alpha$ and $\gamma$ for this class of polymers, are determined by two parameters, $t$, the monomer hopping matrix element (one quarter the bandwidth, $B$ ), and $V$, the exciton excitation energy $\left(\simeq E_{g}+2 t \simeq E_{g}+B / 2\right)$. In addition the monomer length $R_{0}$ plays an obvious role. The factor $a \approx \sqrt{2}$ is nearly constant and hence has little effect. The simplicity of this model plus the relation of the theoretical quantities $(t, V)$ to spectroscopic observables $\left(E_{g}, B\right)$ should make these relations useful in providing insights for designing and developing new materials.

The saturation length depends only on one parameter, $\eta=t / V$. Inclusion of Coulomb interactions and finite frequency (far from resonance) leads to little change in the saturation length. These results lead to the simple empirical formulas (30) and (31) relating saturation length to $\eta$. Thus to design new materials with longer saturation length and with larger magnitudes for $\alpha$ and $\gamma$, one should increase $t$ and/or decrease $V$.

To predict $\alpha$ and $\gamma$ for other materials we need only estimate $t$ and $V$ and substitute into Eqs. (21) and (23). The value of $t$ can be estimated from the conduction bandwidth (27), $B \approx 4 t$. Given $t$, the value for $V$ can be estimated from the energy gap Eq. (28), $V=E_{g}+2 t$. These values of $B$ and $E_{g}$ can be obtained either from experiment or theory. To illustrate the use of this approach we predict in Table III the values of $\alpha, \gamma$, and the saturation lengths $\left(L_{\alpha}, L_{\gamma}\right)$ for a number of polymers (see Fig. 6) based on published values of the bandwidth and band gap.

\section{Comparisons to other theory}

The hyperpolarizability of polymers has been studied in a number of previous papers.
Flytzanis et al. ${ }^{18}$ used the one-electron tight-binding method (extended Hückel) to get band structures of infinite conjugated polymers as a function of the nearest neighbor hopping integral $\beta_{1}$ and $\beta_{2}$ (for two different bond distances). When $\beta_{1} \neq \beta_{2}$, this leads to two bands of states, one occupied and one empty. They considered properties as a function of the bond alternation parameters a Polydiacetylene(Acetyleneic)<smiles>C=C(P)C#CC1=C(C)CC1</smiles>

c Polyparaphenylene vinylene<smiles>CC=Cc1ccc(C(C)(C)C)cc1</smiles>

e Polythiophene vinylene<smiles>CC=Cc1ccc(C(C)(C)C)s1</smiles>

9 Polymethineimine<smiles>CC(C)=NC(C)(C)C</smiles>

i Polyacetylene (trans)<smiles>C=CC=CCC(C)(C)C</smiles>

b Polyparaphenylene

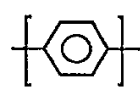

d Polyprrole<smiles>CC(C)(C)c1ccc(C(C)(C)C)[nH]1</smiles>

f Polyvinylene sulfide<smiles>CCCSC=C(C)C</smiles>

in Polybenzothiophene

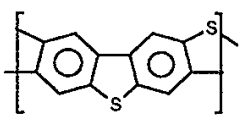

j Polythiophene

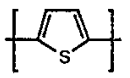

FIG. 6. Repeating units for polymers of Table III. 


$$
N_{d}=\frac{\beta_{2}+\beta_{1}}{\beta_{2}-\beta_{1}}
$$

and used the sum-over-state method to get an expression for the hyperpolarizability of the infinite chain in terms of $N_{d}$. They showed that the hyperpolarizability is quite sensitive to bond alternation, $\gamma \sim N_{d}^{6}$. Such calculations do not include the Coulomb interactions. They did not relate these results to the properties of finite chains and did not discuss the saturation as a function of length.

Garito and co-workers ${ }^{19}$ analyzed the results of extensive configuration interactions (all single and double excitations) based on CNDO/S semiempirical molecular orbitals. These calculations were carried out on polyenes up to $\mathrm{C}_{18} \mathrm{H}_{20}$ ( 9 double bonds). They showed that $y$ is dominated by transitions such as $1^{1} A_{g} \rightarrow 1{ }^{1} B_{u} \rightarrow 5{ }^{1} A_{g} \rightarrow{ }^{1} B_{u} \rightarrow 1{ }^{1} A_{g}$ and $1^{1} A_{g} \rightarrow 1{ }^{1} B_{u} \rightarrow 1{ }^{1} A_{g} \rightarrow 1{ }^{1} B_{u} \rightarrow 1{ }^{1} A_{g}^{g}$ (for hexatriene). These correspond to the strong $\pi$ to $\pi^{*}$ charge transfer excitation $\left(1^{1} B_{1}\right)$ and to a valence-bond-like excited state $\left(5^{1} A_{g}\right)$, which in the molecular orbital framework involves a large number of double excitations. They found that $\gamma_{x x x x}(-3 \omega, \omega, \omega, \omega)$ is proportional to $L^{3.5}$ up to 9 double bonds with no indication of saturation. Because of cost it was not possible to go beyond 9 double bonds to examine the saturation behavior. They assumed that $\gamma$ will saturate at 50 $100 \AA$, which is in good agreement with our result (24 double bonds, 48 carbons or $58 \AA$, see Table III). The charge transfer excitations $\left(1^{1} B_{1}\right)$ are explicitly included in our treatment. The VB excited state $\left(5^{1} A_{g}\right)$ is included in a full VB description but is only approximately accounted for in our treatment.

Dupuis and co-workers ${ }^{20}$ calculated the static polarizability and second hyperpolarizability tensors of the polyenes $\mathrm{C}_{4} \mathrm{H}_{6}$ to $\mathrm{C}_{22} \mathrm{H}_{24}$ via ab initio coupled-perturbed HartreeFock theory. They discussed the chain length dependence of the properties. They did not find saturation; however, based on the decreasing chain length dependence, they suggested that $\alpha_{x x} / N$ and $\gamma_{x x x x} / N$ should eventually approach asymptotic values.

Previous calculations of saturation lengths include Beratan et al..$^{5}$ for polyenes and Brédas et al. ${ }^{6}$ for oligothiophenes. Beratan used a tight-binding method to calculate the length dependence of the second hyperpolarizability for polyene. The hyperpolarizability increased rapidly for small $N$ and then, after about $10-15 C_{2}$ repeating units (20-30 carbons), approaches an asymptotic value. (We find saturation for $12 C_{4}$ repeating units or 48 carbons.) Brédas used the one-electron tight-binding method with a sum-over-states formalism for oligothiophene chains. They predicted that the saturation length is about 50 carbons ( 25 monomers) which is much larger than experiment ( 14 carbons, 7 monomers) and our calculation (17 carbons, 8.6 monomers).

Another recent approach to predicting NLO properties is by Mukamel and co-workers ${ }^{14}$ who studied polyacetylene chains without donor-acceptor groups. They used the Pariser-Parr-Pople (PPP) Hamiltonian and solved the equations of motion for pairs of electrons and holes by assuming pairs of different types are decoupled. This approach describes the NLO properties in terms of the motion of electron-hole, electron-electron, and hole-hole pairs and is much simpler than a standard sum over molecular orbital states approach. They predicted the saturation length of $\gamma$ for polyacetylene is about 21 double bonds which agrees with our calculation ( 24 double bonds). VB-CTE further simplifies the calculation by considering only the electron-hole pairs (excitons) since the electron-electron and hole-hole pairs are much higher in energy.

\section{SUMMARY}

By focusing solely on electron-hole pairs, we constructed a simple Hamiltonian for a conjugated polymer chain and solved for the properties using perturbation theory. Analytical formulas are derived for $\alpha$ and $\gamma$ in terms of just two parameters, $V$ and $t$, plus the overall monomer length, $R_{0}$. These formulas predict the experimental values of $\alpha$ and $\gamma$ within an order of a magnitude and show that $\alpha$ and $\gamma$ can be increased by (i) decreasing the cost of creating an exciton $(V)$ and (ii) once it is created by increasing the energy gain $(t)$ of delocalizing it over many repeat units.

We find that the saturation length is determined by only single parameter, the ratio of $t$ (the hopping matrix element between nearest monomers) and $V$ (the exciton excitation energy). These two parameters are obtained easily from experiment or theory. In addition we obtained the simple empirical formulas (30) and (31) for predicting saturation lengths. These results should be valuable for designing new materials and for interpreting experiments.

\section{APPENDIX A: EVALUATION OF MATRIX ELEMENTS}

To illustrate the evaluation of Eqs. (8) and (9), consider the butadiene monomer (Fig. 4), where

$$
\begin{aligned}
\Theta_{p}^{\mathrm{LUMO}}=\sum_{i=1}^{4} C_{i p}^{\mathrm{LUMO}} \chi_{i}^{p}= & 0.602 \chi_{1}^{p}-0.372 \chi_{2}^{p} \\
& -0.372 \chi_{3}^{p}+0.602 \chi_{4}^{p}, \\
\Theta_{p}^{\mathrm{HOMO}}=\sum_{i=1}^{4} C_{i p}^{\mathrm{HOMO}} \chi_{i}^{p}= & 0.602 \chi_{1}^{p}+0.372 \chi_{2}^{p} \\
& -0.372 \chi_{3}^{p}-0.602 \chi_{4}^{p},
\end{aligned}
$$

and $\chi_{1}^{p}$ are the four atomic orbitals on monomer $p$.

For electron hops this leads to

$$
\begin{aligned}
-t=\left\langle\phi_{p q}|\mathscr{B}| \phi_{p, q+1}\right\rangle & =\left\langle\theta_{p}^{\mathrm{HOMO}} \theta_{p+q}^{\mathrm{LUMO}} \mid \not \nexists \theta_{p}^{\mathrm{HOMO}} \theta_{p+q+1}^{\mathrm{LUMO}}\right\rangle \\
& =\left\langle\theta_{p+q}^{\mathrm{LUMO}}|h| \theta_{p+q+1}^{\mathrm{LUMO}}\right\rangle \\
& =\left\langle 0.602 \chi_{4}^{p}|h| 0.602 \chi_{\mathrm{I}}^{p+1}\right\rangle \\
& =-0.36 \beta,
\end{aligned}
$$

where $\beta$ is the resonance integral between two adjacent $s p^{2}$ carbon centers. Thus Eq. (6) becomes

$$
t=0.36 \beta \text {. }
$$

For excitation to adjacent sites we obtain 


$$
\begin{aligned}
& \left\langle\phi_{p 0}|\cdot| \cdot \mid \phi_{p 1}\right\rangle \\
& =\left\langle\theta_{p}^{\mathrm{HOMO}} \theta_{p}^{\mathrm{HOMO}} \mid \frac{\left(\theta_{p}^{\mathrm{HOMO}} \theta_{p+1}^{\mathrm{LUMO}}+\theta_{p+1}^{\mathrm{LUMO}} \theta_{p}^{\mathrm{HOMO}}\right)}{\sqrt{2}}\right\rangle \\
& =\frac{2}{\sqrt{2}}\left\langle\theta_{p}^{\mathrm{HOMO}}|h| \theta_{p+1}^{\mathrm{LUMO}}\right\rangle \\
& =\frac{-2}{\sqrt{2}} * 0.36 \beta=-\sqrt{2} t .
\end{aligned}
$$

Similarly

$$
\left\langle\phi_{p 0}|\not| \not \mid \phi_{p,-1}\right\rangle=\sqrt{2} t
$$

Thus Eq. (7) leads to

$$
a=\sqrt{2} \text {. }
$$

More generally we can write the coefficient of the outer orbital on $\theta^{\mathcal{U M O} O}$ as $C_{l}$ and on $\theta^{\mathrm{HOMO}}$ as $C_{h}$ to obtain

$$
\begin{aligned}
& t=C_{l}^{2} \beta, \\
& a t=\sqrt{2} C_{l} C_{h} \beta .
\end{aligned}
$$

Thus

$$
a=\frac{\sqrt{2} C_{h}}{C_{l}} .
$$

If $C_{l}=C_{h}$, then

$$
a=\sqrt{2} \text {. }
$$

A simple approximation is $C_{l} \approx 1 / \sqrt{ } M$ and $C_{h} \approx 1 / \sqrt{ } M$, where $M$ is the number of atoms ( $M=4$ for butadiene) so that

$$
a \approx \sqrt{2} \text {. }
$$

Even if $C_{l}=2 C_{h}$ we obtain $a=0.5 \sqrt{2}$.

\section{APPENDIX B: APPLICATION OF PERTURBATION THEORY}

Using Eq. (23), the ground state energy $E^{0}$ can be decomposed as follows: ${ }^{11}$

$$
E^{0}=\sum_{i=0}^{\infty} \Delta_{i}
$$

where

$$
\begin{aligned}
& \Delta_{0}=E_{0}, \\
& \Delta_{1}=\langle 0|W| 0\rangle, \\
& \Delta_{2}=\sum_{j \neq 0} \frac{|\langle 0|W| j\rangle|^{2}}{E_{0}-E_{j}}, \\
& \Delta_{3}=\sum_{j \neq 0}\langle 0|W| j\rangle\langle j \mid 0 ; 2\rangle, \\
& \Delta_{n}=\sum_{j \neq 0}\langle 0|W| j\rangle\langle j \mid 0 ; n-1\rangle-\sum_{\nu=2}^{n-2} \Delta_{\nu}\langle 0 \mid 0 ; n-\nu\rangle .
\end{aligned}
$$

Here we use the notation

$$
\begin{aligned}
\langle 0 \mid 0 ; 1\rangle= & 0, \\
\langle l \mid 0 ; 1\rangle= & \frac{\langle l|W| 0\rangle}{E_{0}-E_{l}} \quad(l \neq 0), \\
\langle 0 \mid 0 ; 2\rangle= & -\frac{1}{2} \sum_{j}\langle j \mid 0 ; 1\rangle^{2}, \\
\langle l \mid 0 ; 2\rangle= & -\sum_{j} \frac{\langle l|W| j\rangle\langle\mid 0 ; 1\rangle}{E_{l}-E_{0}} \\
& -\frac{\langle l|W| 0\rangle\langle 0|W| 0\rangle}{\left(E_{l}-E_{0}\right)^{2}}(l \neq 0), \\
\langle 0 \mid 0 ; n\rangle= & -\frac{1}{2} \sum_{\nu=1}^{n-1} \sum_{j}\langle j \mid 0 ; \nu\rangle\langle j \mid 0 ; n-\nu\rangle, \\
\langle l \mid 0 ; n\rangle= & -\sum_{j} \frac{\langle l|W| j\rangle\langle\mid 0 ; n-1\rangle}{E_{l}-E_{0}} \\
& +\sum_{\nu=1}^{n-1} \frac{\Delta_{\nu}\langle l \mid 0 ; n-\nu\rangle}{E_{l}-E_{0}} \quad(l \neq 0) .
\end{aligned}
$$

Substituting Eqs. (B1), (B2), and (B3) into Eqs. (19)-(20) leads to ${ }^{16}$

$$
\begin{aligned}
& \alpha_{z z}=\frac{4 a^{2} e^{2} R_{0}^{2} \eta^{2}}{V} \sum_{i=0}^{\infty} A_{2 i} \eta^{2 i}, \\
& \beta_{z z z}=0, \\
& \gamma_{z z z z}=\frac{8 a^{2} e^{4} R_{0}^{4} \eta^{2}}{V^{3}} \sum_{i=0}^{\infty} G_{2 i} \eta^{2 i},
\end{aligned}
$$

where

$\eta=\frac{t}{V}$

$A_{0}=1$,

$A_{2}=11-8 a^{2} \approx-5$,

$A_{4}=80-144 a^{2}+60 a^{4} \approx 32$,

$A_{6}=490-1488 a^{2}+1456 a^{4}-448 a^{6} \approx-396$,

$A_{8}=2730-1194 a^{2}+19260 a^{4}-13440 a^{6}+3360 a^{8}$

$\approx 6608$,

$A_{10}=14322-82776 a^{2}+189816 a^{4}-214720 a^{6}$

$$
+118800 a^{8}-25344 a^{10} \approx-19934 \text {, }
$$

$G_{0}=1$,

$G_{2}=57-18 a^{2} \approx 21$,

$G_{4}=1 \quad 170-1104 a^{2}+224 a^{4} \approx-142$,

$G_{6}=15403-27390 a^{2}+14760 a^{4}-2400 a^{6} \approx 463$, 


$$
\begin{aligned}
G_{8}= & 157564-433488 a^{2}+420816 a^{4}-168960 a^{6} \\
& +23760 a^{8} \approx 2332, \\
G_{10}= & 1368796-5233928 a^{2}+7684768 a^{4} \\
& -5372640 a^{6}+1777776 a^{8}-224224 a^{10} \\
\approx & -71860, \\
G_{12}= & 10602592-52717056 a^{2}+106140480 a^{4} \\
& -110176640 a^{6}+61850880 a^{8} \\
& -17751552 a^{10}+2050048 a^{12} \approx 1084778 .
\end{aligned}
$$

In each case we have substituted $a \approx \sqrt{2}$.

The above coefficients are for long chains. If the chain length is small, the coefficients are different. For perturbation theory in which the $V_{n}$ are not constant, we find that the expressions for $A_{0}, G_{0}$ are just functions of $t, V_{1}$, the expressions for $A_{2}, G_{2}$ are functions of $t, V_{1}, V_{2}$, the expressions for $A_{4}, G_{4}$ are functions of $t, V_{1}, V_{2}, V_{3}$, etc. When the order of expansion increase, the coefficients in the Hamiltonian matrix involved in the expression expand gradually along the diagonal. Thus we can consider that $A_{0}, G_{0}$ are contributions from charge transfer to the nearest neighbor site, $A_{2}, G_{2}$ are contributions from charge transfer to the second nearest neighbor sites, etc. The convergence length of the polynomial should correspond to the saturation length. In order to get the accurate values of $\alpha_{z z}, \gamma_{z z z z}$ from the polynomial, we must calculate to the $N$ terms where $2 N$ is the saturation length.

\section{APPENDIX C: THE EVALUATION OF $\boldsymbol{t}$ AND $\boldsymbol{v}$}

We can estimate the values of $t$ and $V$ from the band gap and the conduction bandwidth. The eigenvalues $E$ of the Hamiltonian matrix (4) satisfy the difference equation

$$
-t b_{n-1}+\left(V_{n}-E\right) b_{n}-t b_{n+1}=0 \text {. }
$$

When $n$ is large, $V_{n}$ approaches the constant value IP-EA. In this case Eq. (C1) becomes a homogeneous linear difference equation ${ }^{17}$ with the solutions

$$
b_{n}=C_{1} \lambda_{1}^{n}+C_{2} \lambda_{2}^{n} \text {, }
$$

where $\lambda_{1}$ and $\lambda_{2}$ are solutions of

$$
\begin{aligned}
& -t \lambda^{2}+(V-E) \lambda-t=0, \\
& \lambda=\frac{V-E}{2 t} \pm \sqrt{\left(\frac{V-E}{2 t}\right)^{2}-1 .}
\end{aligned}
$$

This leads to complex conjugate roots (oscillating $b_{n}$ ) when

$$
|V-E|<2 t \text {. }
$$

These states Eq. (C5) correspond to the conduction states, leading to a bandwidth for the conduction band of

$$
B=4 t \text {. }
$$

The upper bound of the conduction band is $V+2 t$ and the lower bound is $V-2 t$. For small $n, V_{n}$ is smaller than $V$ which will decrease the lower bound, $V-2 t$. Coupling between the ground state and excited states decreases the ground state energy by about $-\left(2 a^{2} t^{2} / V\right)$. From the full calculation [diagonalizing Eq. (4)], we can write the band gap approximately as

$$
E_{g} \approx V-2 t+\frac{a^{2} t^{2}}{V}
$$

or more simply

$$
E_{g}=V-2 t \text {. }
$$

Solving Eq. (C7) for $V$ leads to

$$
V \approx\left(\frac{1}{2} E_{g}+t\right)+\sqrt{\left(\frac{1}{2} E_{g}+t\right)^{2}-a^{2} t^{2}}
$$

\section{ACKNOWLEDGMENTS}

We wish to thank Dr. Seth Marder and Professor Julie Kornfield of Caltech for helpful suggestions. The research was funded by NSF (CHE 91-100289). The facilities of the MSC are also supported by grants from DOE-AICD, NSFACR, Allied-Signal Corp., Asahi Chemical, Asahi Glass, BP America, Chevron, BF Goodrich, Teijin Chemical, Xerox, and Beckman Institute.

\footnotetext{
${ }^{1}$ Introduction to Nonlinear Optical Effects in Molecules and Polymers, edited by P. N. Prasad and D. J. Williams (Wiley, New York, 1991).

${ }^{2}$ S. R. Marder, D. N. Beratan, and L.-T. Cheng, Science 252, 103 (1991).

${ }^{3}$ L. T. Cheng, W. Tam, S. H. Stevenson, G. R. Meredith, G. Rikken, and S. R. Marder, J. Phys. Chem. 95, 10631 (1991).

${ }^{4}$ H. Thinpont, G. L. A. Rikken, E. W. Meijer, W. ten Hoeve, and H. Wynberg, Phys. Rev. Lett. 65, 2141 (1990).

${ }^{5}$ D. N. Beratan, J. N. Onuchic, and J. W. Perry, J. Phys. Chem. 91, 2696 (1987).

${ }^{6}$ Z. Shuai and J. L. Brédas, Phys. Rev. B 44, 5962 (1991).

${ }^{7}$ J. R. Heflin, K. Y. Wong, O. Zamani-khamiri, and A. F. Garito, Phys. Rev. B 38, 1573 (1988).

${ }^{8}$ B. I. Greene, J. Orenstein, and S. Schmitt-Rink, Science 247, 679 (1990),

${ }^{9}$ B. J. Orr and J. F. Ward, Mol. Phys. 20, 513 (1971).

${ }^{10}$ J. A. Pople and S. H. Walmsley, Faraday Soc. Cont. 15, 441 (1962).

${ }^{11}$ E. M. Corson, Perturbation Methods in the Quantum Mechanics of n-Electron Systems (Hafner, New York, 1951).

${ }^{12}$ P. Tavan and K. Schulten, Phys. Rev. B 36, 4337 (1987).

${ }^{13}$ W.-S. Fann, S. Benson, J. M. J. Madey, S. Etemad, G. L. Baker, and F. Kajzar, Phys. Rev. Lett. 62, 1492 (1989).

${ }^{14}$ S. Mukamel and H. X. Wang, Phys. Rev. Lett. 69, 65 (1992).

${ }^{15}$ Handbook of Conducting Polymers, edited by Terje A. Skotheim (Marcel Dekker, New York, 1986), Vol. 2, p. 859.

${ }^{16}$ Mathematica, Version 2.0, Wolfram Research, Inc., Champaign, Iilinois.

${ }^{17} \mathrm{C} . \mathrm{H}$. Richardson, An Introduction to the Calculus of Finite Differences (van Nostrand, New York, 1954), p. 108.

${ }^{18}$ M. Zhao, B. P. Singh, and P. N. Prasad, J. Chem. Phys. 89, 5535 (1988); G. P. Agrawal, C. Cojan, and C. Flytzanis, Phys. Rev. B 17, 776 (1978); C. Cojan, G. P. Agrawal, and C. Flytzanis, ibid. 15, 909 (1977).

${ }^{19} \mathrm{~J}$. R. Heflin and A. R. Garito, Nonlinear Optical Materials, edited by H. Kuhn and J. Robillard (Chemical Rubber, Boca Raton, 1992).

${ }^{20}$ G. J. B. Hurst, M. Dupuis, and E. Clementi, J. Chem. Phys. 89, 385 (1988); J. Zyss, ibid. 70, 3333 (1979).

${ }^{21}$ C. P. de Melo and R. Sibey, J. Chem. Phys. 88, 2558 (1988).
} 